\title{
Effet de l'insertion des plantes de couverture sur la productivité du système de culture à base de maïs dans le cadre de l'intégration agriculture-élevage
}

\author{
Fagaye SISSOKO $^{1 *}$, Amadou TRAORE ${ }^{1}$, Sidiki DIARRA $^{2}$ et Mamadou TRAORE ${ }^{2}$ \\ IInstitut d'Economie Rurale (IER), Programme coton, Sikasso, Mali. \\ ${ }^{2}$ Institut d'Economie Rurale (IER), Programme coton, Sotuba, Mali. \\ *Auteur correspondant; E-mail : fagaye_sissoko@yahoo.fr; Tél : +22366798171 /+2237703 3540
}

\section{REMERCIEMENTS}

Les auteurs remercient la Coopération Brésilienne et les pays partenaires du Projet Coton C4+Tchad. Ce travail a été soutenu financièrement par l'Agence Brésilienne de Coopération (ABC) à travers l'Ambassade Brésilienne au Mali.

\section{RESUME}

En zone soudano-sahélienne, la productivité des cultures est limitée par les effets néfastes du changement climatique et la pauvreté des sols. L'insertion des plantes de couverture dans les systèmes de production pourrait être une alternative d'amélioration des rendements et de la biomasse. Pour atteindre cet objectif, la pratique conventionnelle de la culture du maïs a été comparée pendant cinq années (2014-2018), à quatre systèmes de culture associant des plantes de couverture. Le dispositif expérimental utilisé a été un bloc de Fisher avec 6 traitements en 4 répétitions. Les résultats ont montré que l'insertion du Cajanus cajan, du Stylosanthes hamata, du Brachiaria ruziziensis et Mucuna cochinchinensis dans un système de culture à base du maïs permet d'améliorer la production de biomasse fourragère sans négativement affecter son rendement. Utilisée dans la supplémentation des animaux, la biomasse produite peut nourrir pendant 90 jours 7 unités de bétail tropical (UBT) en culture pure du maïs et 8 à 13 unités de bétail tropical (UBT) en fonction du type de plantes de couverture. Dans le cadre de la production fumure organique, les mêmes tendances de variations ont été obtenues en fonction des systèmes de culture. L'insertion des plantes de couverture est un élément intégrateur agricultureélevage.

(C) 2020 International Formulae Group. All rights reserved.

Mots clés : Changement climatique, maïs, légumineuse, biomasse fourragère, zone Soudano-sahélienne, Mali

\section{Cover crop insertion effect on productivity of maize-based cropping system in the context of crop-livestock integration}

\begin{abstract}
In Sudano-Sahelian zone, crop productivity is limited by climate change effect and poor soils. Inserting cover crops into production systems could be an alternative to improve yields and biomass. To achieve this goal, conventional practice of maize cultivation was compared over a five-year period (2014-2018) with four cropping systems using cover crops. The experimental design used was a Fisher block with 6 treatments in 4 replicates. The results showed that the insertion of Cajanus cajan, Stylosanthes hamata, Brachiaria ruziziensis and Mucuna
\end{abstract}


cochinchinensis in a maize-based cropping system improves biomass production without negatively affecting its yield. Used in animal supplementation, the biomass produced can feed 7 Tropical Livestock Units (UBT) of pure maize crop and 8 to 13 UBT for 90 days, depending on the type of cover crop. In the case of organic manure production, the same variations were obtained depending on cropping systems. The insertion of cover crops in cropping system is an integrating agriculture-livestock component.

(C) 2020 International Formulae Group. All rights reserved.

Keywords: Climate change, maize, legumes, fodder biomass, fodder biomass, Sudano-Sahelian zone, Mali.

\section{INTRODUCTION}

Depuis plusieurs décennies, l'intégration de l'élevage dans les exploitations des agriculteurs, est un thème récurrent du développement agricole, à cause de la dégradation des sols et de la réduction des espaces pastoraux (Awono et Havard. 2001). En plus de la baisse de la fertilité des sols, les pratiques de gestion inappropriées des sols et cultures, les dégâts des ravageurs et les effets néfastes du changement climatique, entraînent la baisse de rendements des cultures. Cette situation a eu comme impact direct, l'insécurité alimentaire, la réduction des revenus des exploitations, l'exode rural et les conflits entre agriculteurs et éleveurs devenus de nos jours, préoccupants pour les acteurs des différentes filières (Deveze et Halley des Fontaines, 2005).

Concernant la dégradation des sols, les causes majeures rapportées sont, entre autres, le rythme élevé de la croissance démographique qui se manifeste par la destruction du couvert végétal et les terres arables, la baisse de la fertilité des sols, la surexploitation des terres, l'extension des superficies cultivées, la réduction des pâturages ainsi que les superficies des jachères et la durée de leur cycle (Odru, 2013 ; Fleury, 2016). Dans le cadre de la restauration de la fertilité des sols, les systèmes de culture se caractérisaient dans un passé récent, par un temps de mise en culture de trois à cinq ans, alternant avec un temps de jachère qui dépassait généralement dix ans (Balarabé, 2012; Koné, 2013). De nos jours, la restauration de la fertilité des sols qui se fait à travers les relations de complémentarité entre éleveurs transhumants et agriculteurs suivant des " contrats de fumure contre résidus de récolte", a tendance à disparaître (Thibaud et
François, 2010 ; Lemaire, 2014). Ainsi, les agro-éleveurs adoptent alors, l'une des deux alternatives pour l'élevage qui sont, soit son intégration dans l'exploitation, soit l'éloignement des troupeaux de l'habitat. Dans une étude conduite par Bénagabou et al. (2017) au Burkina Faso, l'ensemble des exploitations étudiées étaient propriétaires d'animaux d'élevage et produisaient de la fumure organique entre 1,4 à 7,9 t MS. Cette production de fumure organique est largement insuffisante car ne permet de couvrir qu'entre 9 et $56 \%$ des besoins des sols cultivés (FAO, 2015).

Pour couvrir les besoins alimentaires d'une population et d'un cheptel en pleine croissance, de nombreuses technologies d'intégration agriculture-élevage ont été développées et introduites en milieu paysan (Blanchard, 2010) ; Vall et al., 2012). Parmi ces technologies, l'insertion des plantes de couverture dans les systèmes de production peut être une solution alternative d'amélioration des rendements des cultures et de la production fourragère pour couvrir les besoins nutritionnels des animaux en saison sèche pendant laquelle les aliments deviennent rares. Selon Sissoko et Autfray (2007), cette technologie permet de diminuer l'effet de l'érosion (éolienne et hydrique) et d'améliorer la production de la biomasse fourragère ainsi que les rendements du coton et du sorgho de 10 à $20 \%$ dans les zones de pluviométrie entre 900 à $1000 \mathrm{~mm}$.

L'objectif de cette étude est de contribuer à la gestion durable de la productivité du système de culture à base de maïs à travers l'insertion des plantes de couverture pour la supplémentation des animaux. 


\section{MATERIEL ET METHODES}

\section{Site d'étude}

Cette étude a été menée en station au Centre Régional de Recherche Agronomique (CRRA) de Sotuba situé dans la commune I du district de Bamako sur une altitude de $320 \mathrm{~m}$ entre la latitude $12^{\circ} 66^{\prime} 38^{\prime \prime}$ 'Nord et la longitude de 7०92'22" Ouest. Le climat est de type soudano sahélien avec deux saisons dont une saison pluvieuse de juin à octobre et une saison sèche de novembre à mai. La pluviosité moyenne des 10 dernières années, est de 920 $\mathrm{mm}$ en 70 jours avec une grande variabilité constatée pendant le mois de juin; ce qui retarde souvent la mise en place des cultures de la première décade de Juin à la deuxième décade de Juillet (Figure 1).

Sissoko et al. (2020) ont montré que les sols de la station de Sotuba sont de types ferrugineux tropicaux avec un $\mathrm{pH}$ de 6,1 à l'horizon $0-20 \mathrm{~cm}$, pauvres en $\mathrm{N}$ total $(0,03$ $\mathrm{g} / \mathrm{g}$ ) avec une capacité d'échange cationique qui était faible $\left(5,1 \mathrm{Cmol}^{+} / \mathrm{kg}\right)$ dans l'horizon $0-20 \mathrm{~cm}$. L'analyse granulométrique effectuée a montré qu'en surface $(0-20 \mathrm{~cm})$ le sol est sablo-limoneux avec 14,6 g/100g d'argile, 37,4 $\mathrm{g} / 100 \mathrm{~g}$ de limon et $47,9 \mathrm{~g} / 100 \mathrm{~g}$ de sable. En profondeur $(20-40 \mathrm{~cm})$, il est limoneux avec $19,4 \mathrm{~g} / 100 / \mathrm{g}$ d'argile, $39,3 \mathrm{~g} / 100 \mathrm{~g}$ de limon et 40,6 g/100g de sable. Le Tableau 1 comporte quelques caractéristiques chimiques et $\mathrm{pH}$ des échantillons de sol étudiés.

\section{Matériel}

Pour la réalisation de cette expérimentation, la variété de maïs utilisée a été SUWAN 1 communément appelée "SOTUBAKA" (quelques caractéristiques de la variété utilisée sont données dans le Tableau 2). Les espèces de plantes de couverture utilisées sont les suivantes: Brachiaria ruziziensis, Stylosanthes hamata, Cajanus cajan et Mucuna cochinchinensis. $\mathrm{La}$ fertilisation minérale du maïs a été faite avec l'engrais céréale (15-15-15) et l'urée (46\% de $\mathrm{N})$. L'insecticide utilisé a été la matière active Emamectine benzoate.

\section{Méthodes \\ Dispositif expérimental}

Le dispositif expérimental utilisé a été le bloc de Fisher randomisé avec 6 traitements en 4 répétitions. Les traitements ont été : $(i)$ maïs semé directement sur sol nu sans labour (T1), (ii) maïs associé au Brachiaria ruziziensis (T2), (iii) maïs associé au Brachiaria ruziziensis et Stylosanthes hamata (T3), (iv) maïs associé au Brachiaria ruziziensis et Cajanus cajan (T4), (v) maïs associé au Brachiaria ruziziensis et Mucuna cochinchinensis (T5) et (vi) système conventionnel (T6). Seul le traitement T6 a été labouré avant le semis, les autres traitements ont été semés directement. La variété du maïs Sotubaka, d'un cycle végétatif de 115 à 120 jours, a été semée à la dose quantité de 20 $\mathrm{kg} \cdot \mathrm{ha}^{-1}$. L'écartement entre deux lignes de semis a été de $0,80 \mathrm{~m}$ et celui entre deux poquets a été de $0,40 \mathrm{~m}$. Le démariage a été fait à 2 plants par poquet. Par contre, les plantes de couverture ont été semées juste après le premier désherbage, 15 jours après la levée du maïs (JAL), en lignes continues (Brachiaria ruziziensis et Stylosanthes hamata) entre deux lignes de maïs ou en poquets (Cajanus cajan et Mucuna cochinchinensis) sur la ligne de semis du maïs avec un espacement de $0,80 \mathrm{~m}$ entre chaque deux poquets. Les quantités doses de chaque semence utilisée sont indiquées dans le Tableau 3.

La superficie de la parcelle élémentaire a été de 217,6 $\mathrm{m}^{2}$ soit $34 \mathrm{~m} \mathrm{x} \mathrm{6,4} \mathrm{m.} \mathrm{L'engrais}$ complexe céréale (15-15-15) a été apporté 15 jours après la levée (JAL) à la dose de 200 $\mathrm{kg} \cdot \mathrm{ha}^{-1}$ sur toutes les parcelles comme fumure de fond. Par ailleurs, l'urée a été apportée 45 JAL comme fumure d'entretien, à la dose de $100 \mathrm{~kg} \cdot \mathrm{ha}^{-1}$. Pour éviter d'éventuelle compétition avec les mauvaises herbes pour les nutriments, des désherbages ont été régulièrement effectués sur l'ensemble des parcelles. Le premier a été fait $15 \mathrm{JAL}$, le deuxième 15 jours après le premier et les autres au besoin. De même, l'insecticide Emamectine benzoate a été utilisé (à la dose de 0,5 litre par 
hectare), pour le traitement contre la chenille légionnaire en cas d'infestation.

\section{Collecte des données}

La récolte du maiis a été faite par parcelle utile de $54,4 \mathrm{~m}^{2}$ à la maturation et les graines séchées à l'air libre et pesées pour l'évaluation du poids grain. Par contre, les plantes de couverture ont été fauchées au mois de novembre après la récolte du maïs dans trois carrés de rendement de $1 \mathrm{~m}^{2}$ installés au niveau de chaque parcelle élémentaire. La biomasse produite dans chaque carré de rendement a été pesée puis, un échantillon homogène a été prélevé et pesé parcelle par parcelle. Ainsi, les différents échantillons prélevés ont été placés dans une étuve à $70^{\circ} \mathrm{C}$ pour séchage et évaluation des poids secs de la biomasse des parcellaires. L'estimation du nombre d'Unité de Bétail Tropical(UBT) pouvant être supplémenté a été faite en utilisant les normes suivantes : une UBT est un bovin de $250 \mathrm{~kg}$, sa supplémentation est de $6,25 \mathrm{~kg}$ de Matière sèche (MS)/ jour. La quantité de fumure organique a été estimée à partir des données de la bibliographie. Une UBT en stabulation produit une tonne de MS par an (Sanon et al., 2014 ; Coulibaly et al., 2017).

Les variables mesurées ont porté sur la densité de peuplement du maïs au démariage et à la récolte, la hauteur des plants de maïs, le poids du grain de maïs, la quantité de la biomasse produite, la détermination du nombre d'unité de bétail tropical (UBT) et l'estimation de la quantité de la fumure produite.

\section{Analyse statistique}

L'analyse de variance a été faite à l'aide du logiciel StatBox 6,5 et le test de Newman et Keuls au seuil de $5 \%$ a été utilisé pour la séparation des moyennes.

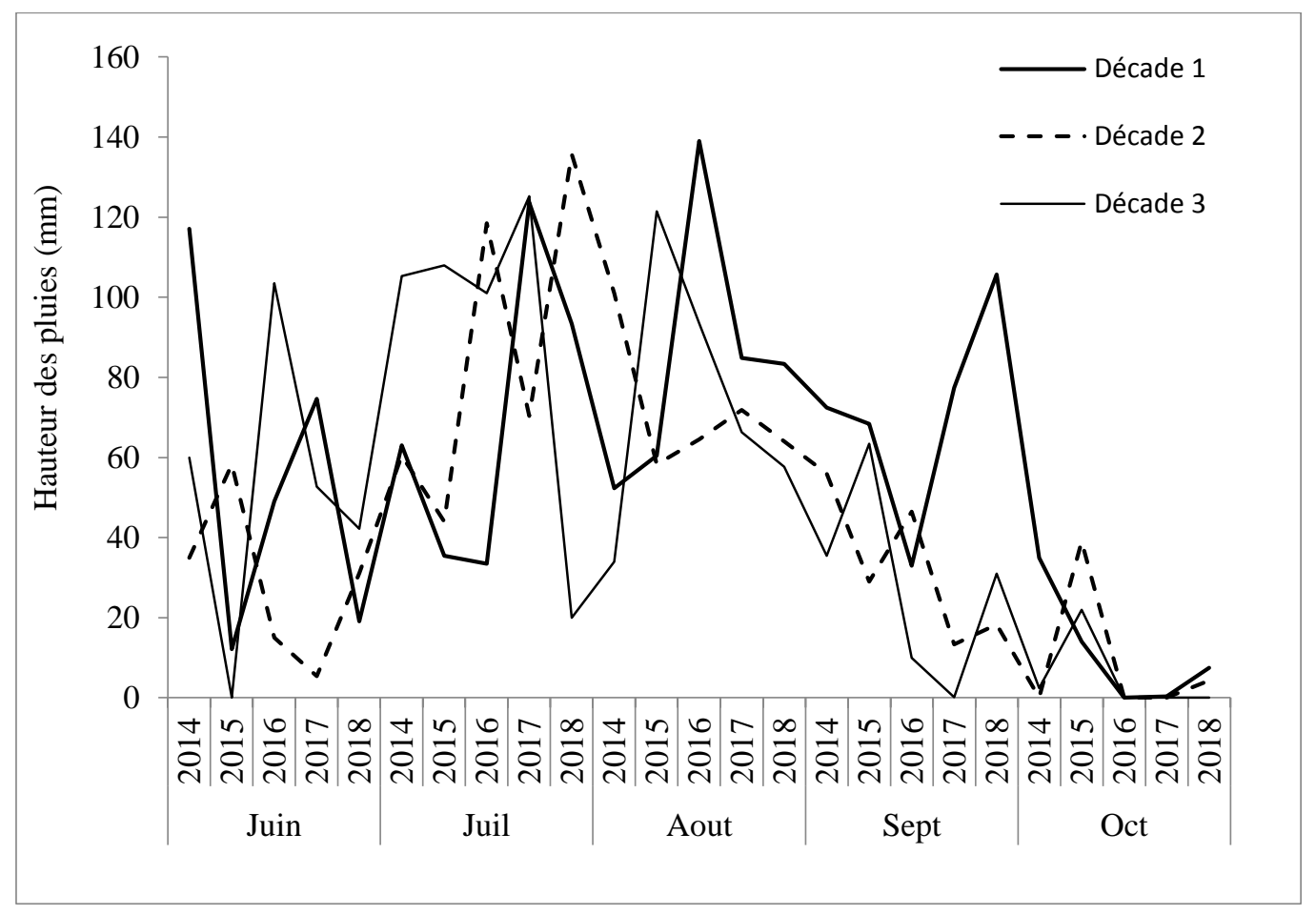

Figure 1 : Hauteurs des pluies recueillies dans la Station de Recherche Agronomique de Sotuba de 2014 à 2018. 
Tableau 1: Quelques caractéristiques chimiques et pH des échantillons de sol du site d'étude.

\begin{tabular}{ccccccccc}
\hline $\begin{array}{c}\mathbf{H} \\
(\mathbf{c m})\end{array}$ & $\begin{array}{c}\mathbf{p H} \\
\mathbf{e a u}\end{array}$ & $\begin{array}{c}\mathbf{N} \\
\mathbf{\%}\end{array}$ & $\begin{array}{c}\mathbf{P} \text { total } \\
\mathbf{p p m}\end{array}$ & $\begin{array}{c}\mathbf{P} \text { ass } \\
\mathbf{p p m}\end{array}$ & $\begin{array}{c}\mathbf{C a} \\
\mathbf{m e ́ q} / \mathbf{1 0 0} \mathbf{g}\end{array}$ & $\begin{array}{c}\mathbf{M g} \\
\mathbf{m e ́ q} / \mathbf{1 0 0} \mathbf{g}\end{array}$ & $\begin{array}{c}\mathbf{K} \\
\mathbf{m e ́ q} / \mathbf{1 0 0} \mathbf{g}\end{array}$ & $\begin{array}{c}\mathbf{C E C} \\
\mathbf{m e ́ q} / \mathbf{1 0 0} \mathbf{g}\end{array}$ \\
\hline $0-20$ & 6,1 & 0,03 & 121,2 & 4,3 & 2,0 & 0,9 & 0,20 & 5,1 \\
\hline $20-40$ & 6,4 & 0,02 & 113,5 & 1,2 & 2,5 & 1,1 & 0,10 & 5,7
\end{tabular}

Légende : $\mathrm{H}$ : Horizon, $\mathrm{N}$ : azote ; P ass : Phosphore assimilable ; Ca : Calcium ; Mg : Magnésium ; K : Potassium ; CEC : Capacité d'Echange Cationique

Tableau 2 : Quelques caractéristiques de la variété de maïs SOTUBAKA utilisée dans l'essai entre 2014-2018.

\begin{tabular}{ll}
\hline Caractéristiques & Variété Sotubaka \\
\hline Cycle & $115-120$ jours \\
Type de graine & Jaune corné \\
Rendement moyen & $5-7$ t.ha $^{-1}$ \\
Hauteur plant & $200-235 \mathrm{~cm}$ \\
Longueur épi & $15-18 \mathrm{~cm}$ \\
Quantité de semence & $15-20 \mathrm{~kg}^{-h^{-1}}$ \\
\hline
\end{tabular}

Tableau 3: Quantités de semences de plantes de couverture utilisées $\left(\mathrm{kg}_{\mathrm{g}} \mathrm{ha}^{-1}\right)$ dans les différents traitements.

\begin{tabular}{lcccc}
\hline Traitements & $\begin{array}{l}\text { Brachiaria } \\
\text { Ruziziensus }\end{array}$ & $\begin{array}{l}\text { Cajanus } \\
\text { cajan }\end{array}$ & $\begin{array}{l}\text { Stylosanthes } \\
\text { hamata }\end{array}$ & Mucuna \\
\hline $\begin{array}{l}\text { T1 : maïs semé directement sur sol nu } \\
\text { sans labour }\end{array}$ & - & - & - & - \\
T2= maïs + Brachiaria ruziziensis & 6 & - & - & - \\
$\begin{array}{l}\text { T3= maïs + Brachiaria ruziziensis + } \\
\text { Stylosanthes hamata }\end{array}$ & 6 & - & 6 & - \\
$\begin{array}{l}\text { T4= maïs + Brachiaria ruziziensis + } \\
\text { Cajanus cajan }\end{array}$ & 6 & 15 & - & - \\
$\begin{array}{l}\text { T5= maïs + Brachiaria ruziziensis + } \\
\text { Mucuna sp }\end{array}$ & 6 & - & - & 20 \\
$\begin{array}{l}\text { T6= maïs seul (exportation des résidus } \\
\text { après la récolte }: \text { système }\end{array}$ conventionnel) & & - & - & - \\
\hline
\end{tabular}


RESULTATS

Effets des systèmes de culture sur le développement végétatif des plantes Effets sur la densité des plants au démariage et à la récolte

Les résultats de comptage du nombre des plants de maïs au démariage et à la récolte de 2014 à 2018 à la Station de Recherche Agronomique (SRA) de Sotuba sont indiqués sur la Figure 2. L'analyse de la variance n'a montré aucune différence significative entre les différents systèmes de culture étudiés. Au démariage et à la récolte, les densités moyennes de plants de maïs associés aux plantes de couverture sont restées statistiquement équivalentes à celles du maïs en culture pure avec résidus conservés à la surface du sol ou exportés des parcelles. Au démariage, les densités ont été de 57800 plants ha $^{-1}$ et de 68900 plants ha ${ }^{-1}$ respectivement pour le semis avec résidus de récolte conservés et semis avec exportation des résidus et l'insertion des plantes de couverture. A la récolte, les densités ont été 51300 plants ha ${ }^{-1}$ et de 52300 plants $^{-1}{ }^{-1}$ respectivement pour les parcelles avec résidus et sans résidus plus plantes de couverture.

D'une manière générale, les nombres moyens des plantes au démariage ont été nettement supérieurs à ceux obtenus à la récolte.

\section{Influence sur la croissance du maïs}

Les résultats des études réalisées en station à Sotuba de 2014 à 2018, ont montré que l'insertion des plantes de couverture dans les systèmes de culture du maïs, est sans effet sur la croissance du maïs (Tableau 4). Les hauteurs moyennes des plants de maïs relevées dans les parcelles semées sur résidus et sans résidus de récolte, sont restées statistiquement similaires à celles obtenues dans les parcelles avec plantes de couverture, à la probabilité de $5 \%$. Par ailleurs, une tendance à la hausse des hauteurs moyennes a été observée en 2018 par rapport à celles enregistrées de 2014 à 2017. Cette tendance s'explique par le semis précoce intervenu dans la première décade de juin 2018 par rapport aux autres années pendant lesquelles les périodes de semis se sont situées entre la deuxième décade de juin et la deuxième décade de juillet à cause des conditions pluviométriques (Figure 1).

\section{Effet de l'insertion des plantes de couverture sur le rendement du maïs}

Les résultats d'évaluation indiqués sur la Figure 3, ont montré que l'insertion du Cajanus cajan, du Stylosanthes hamata ou du Brachiaria ruziziensis comme plantes de couverture dans les systèmes de culture à base du maïs, n'a aucun effet significatif sur les rendements moyens obtenus des différents systèmes de culture testés $(\mathrm{P}>0.05)$. Les rendements obtenus à l'hectare ont été $4066 \mathrm{~kg}$ pour les parcelles avec insertion Brachiaria ruziziensis contre $4723 \mathrm{~kg}$ pour les parcelles ayant abritées le maïs semé sur résidus de récolté. Ces résultats sont similaires avec ceux annuellement reportés en 2014, 2015 et 2016 (Tableau 3). Par contre, en 2017, le meilleur rendement (4861 kg.ha-1) a été obtenu avec l'insertion du Brachiaria ruziziensis et Cajanus cajan $(\mathrm{P}<0.05)$ tandis qu'en 2018 , les meilleurs rendements ont été obtenus avec les traitements sans plantes de couverture (Figure 4).

Effet de l'insertion des plantes de couverture sur la production et l'utilisation de la biomasse fourragère

Evaluation de la production de biomasse fourragère totale

La Figure 4 indique les résultats d'évaluation de l'effet de l'insertion des plantes de couverture sur la production de biomasse fourragère totale incluant les tiges de maïs et les biomasses aériennes des plantes de couverture de 2014 à 2018 en station de Sotuba. L'analyse de ces résultats a révélé que l'insertion de Brachiaria ruziziensis, du Stylosanthes hamata, du Cajanus cajan et du Mucuna $s p$ dans le système de culture à base de maïs, améliore significativement la production de la biomasse fourragère $(P \leq 0,05)$. Le meilleur rendement a été obtenu avec le maïs associé au Brachiaria ruziziensis et Mucuna sp avec un taux d'augmentation de $101 \%$ par rapport à celui du maïs seul, semé sur sol avec résidus de récolte et $76 \%$ sur sol sans résidus. Les rendements estimés avec l'insertion des 
autres plantes de couverture (Brachiaria ruziziensis, Brachiaria ruziziensis et Stylosanthes hamata ou Brachiaria ruziziensis et Cajanus cajan) sont restés nettement inférieurs à celui obtenu avec Brachiaria ruziziensis et Mucuna sp et supérieurs au rendement de biomasse produite avec le semis sur résidus. Les taux moyens d'augmentation obtenus par rapport aux témoins semés sur résidus et sans résidus de récolte, ont été respectivement, de $32 \%$ et $15 \%$ avec l'insertion de Brachiaria ruziziensis seul, $46 \%$ et $26 \%$ avec Brachiaria ruziziensis et Stylosanthes hamata et $60 \%$ et $40 \%$ avec Brachiaria ruziziensis et Cajanus cajan. Par contre, les rendements les plus faibles ont été enregistrés avec le semis du maïs sur résidus de récolte (3487 kg.ha ${ }^{-1}$ ) et sans résidus (3992 kg.ha$\left.{ }^{1}\right)$. Ces résultats sont en adéquation avec les variations annuelles de rendements en biomasse observées en fonction des systèmes de culture entre 2014 et 2018 (Figure 4).

Valorisation de la biomasse fourragère

Dans cette étude, la valorisation des quantités de biomasse fourragère totale produite par système de culture a été déterminée en fonction du nombre d'unité de bétail tropical (UBT) pouvant être supplémenté pendant 90 jours et de la quantité de fumure organique produite pendant cette même période (Tableau 6). L'analyse des résultats d'évaluation a révélé des différences significatives entre les nombres d'UBT estimés avec les six systèmes de culture $(\mathrm{P}<0,05)$. La quantité de biomasse fourragère produite (7029 kg.ha-1) avec l'insertion du Brachiaria ruziziensis et Mucuna $s p$ dans le système de culture du maïs, permet de supplémenter 12 UBT correspondant au plus grand nombre d'UBT pendant 90 jours, par rapport aux autres systèmes. Les productions de biomasse des autres systèmes de culture qui ont été statistiquement similaires (Figure 4), permettent de supplémenter des nombres d'UBT équivalents. Par contre, la faible quantité de biomasse (4954 $\mathrm{kg} \mathrm{ha}^{-1}$ ) qui a été produite par la culture pure du maïs avec exportation des résidus de récolte, permet de supplémenter seulement 7 UBT qui constituent le plus faible nombre.

Dans le cadre de la production de la fumure organique, les mêmes tendances de variations observées avec les nombres moyens d'UBT en fonction des systèmes de culture, ont été également obtenues avec les quantités moyennes de fumure organique produites pendant la même période.

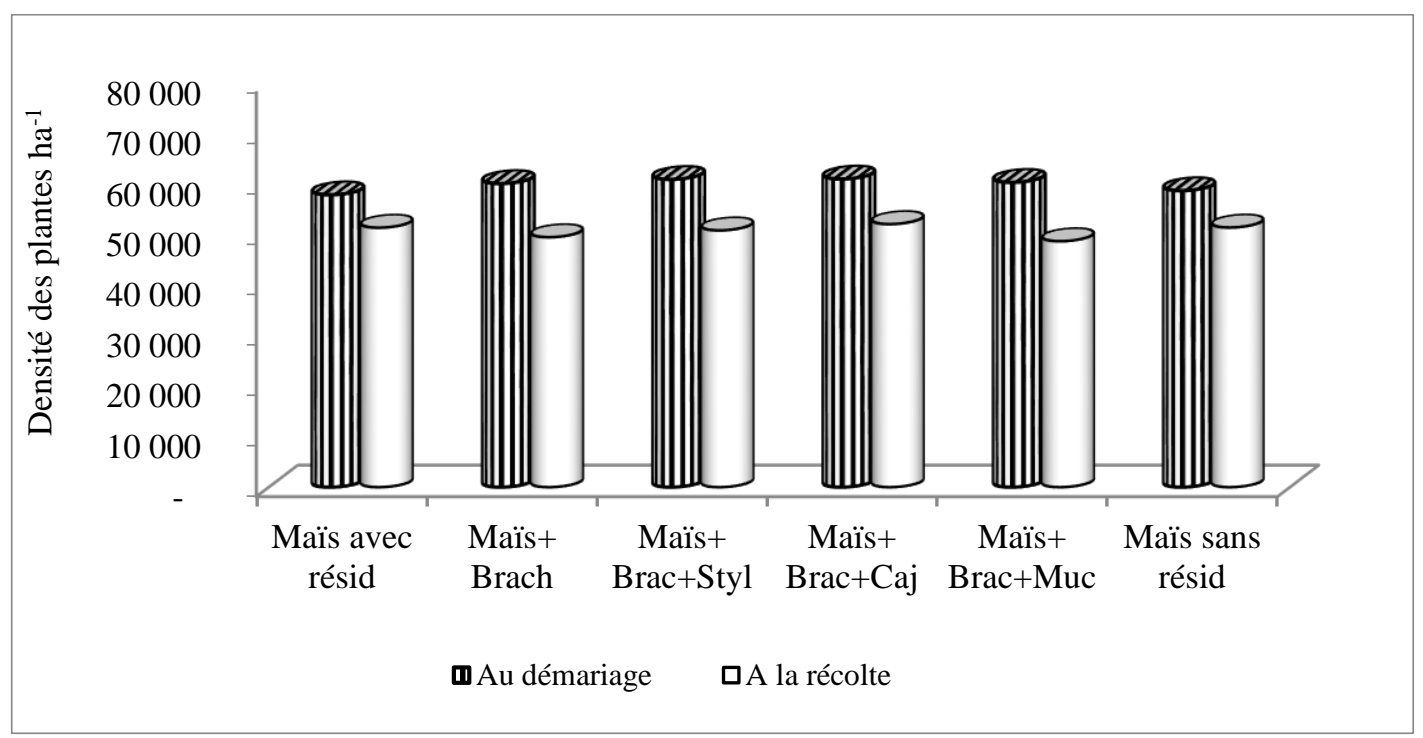

Légende $:$ Résid $=$ résidus de récolte $;$ Brach $=$ Brachiaria ruziziensis $;$ Caj $=$ Cajanus cajan $;$ Muc $=$ Mucuna $s p$

Figure 2 : Nombre moyen des plants de maïs au démariage et à la récolte de 2014 à 2018 dans les différents systèmes de culture en station à Sotuba. 
Tableau 4 : Hauteurs moyennes des plants de maïs de 2014 à 2018 dans les différents systèmes de culture en station à Sotuba.

\begin{tabular}{lcccccc}
\hline \multirow{2}{*}{ Systèmes de culture } & \multicolumn{5}{c}{ Hauteurs moyennes (m) } \\
\cline { 2 - 7 } & $\mathbf{2 0 1 4}$ & $\mathbf{2 0 1 5}$ & $\mathbf{2 0 1 6}$ & $\mathbf{2 0 1 7}$ & $\mathbf{2 0 1 8}$ & Moyennes \\
\hline $\begin{array}{l}\text { Maïs semé directement sur sol sans labour } \\
\text { et sans résidus de récolte }\end{array}$ & 2,46 & 2,54 & 2,50 & 2,40 & 2,62 & 2,50 \\
$\begin{array}{l}\text { Maïs + Brachiaria ruziziensis } \\
\text { Maïs + Brachiaria ruziziensis + }\end{array}$ & 2,35 & 2,60 & 2,48 & 2,47 & 2,63 & 2,51 \\
$\begin{array}{l}\text { Stylosanthes hamata } \\
\text { Maïs + Brachiaria ruziziensis + Cajanus }\end{array}$ & 2,45 & 2,62 & 2,48 & 2,57 & 2,74 & 2,57 \\
cajan & & 2,52 & 2,37 & 2,53 & 2,58 & 2,48 \\
$\begin{array}{l}\text { Maïs + Brachiaria ruziziensis + Mucuna } \\
\text { sp }\end{array}$ & 2,25 & 2,63 & 2,45 & 2,48 & 2,56 & 2,47 \\
$\begin{array}{l}\text { Maïs seul (exportation des résidus après la } \\
\text { récolte : système conventionnel) }\end{array}$ & 2,39 & 2,56 & 2,48 & 2,46 & 2,57 & 2,49 \\
\hline $\begin{array}{l}\text { Ecart type } \\
\text { CV (\%) }\end{array}$ & 0,14 & 0,08 & 0,06 & 0,08 & 0,11 & 0,09 \\
Probabilité & 5,88 & 1,4 & 2,54 & 3,05 & 4,09 & 3,04 \\
\hline
\end{tabular}

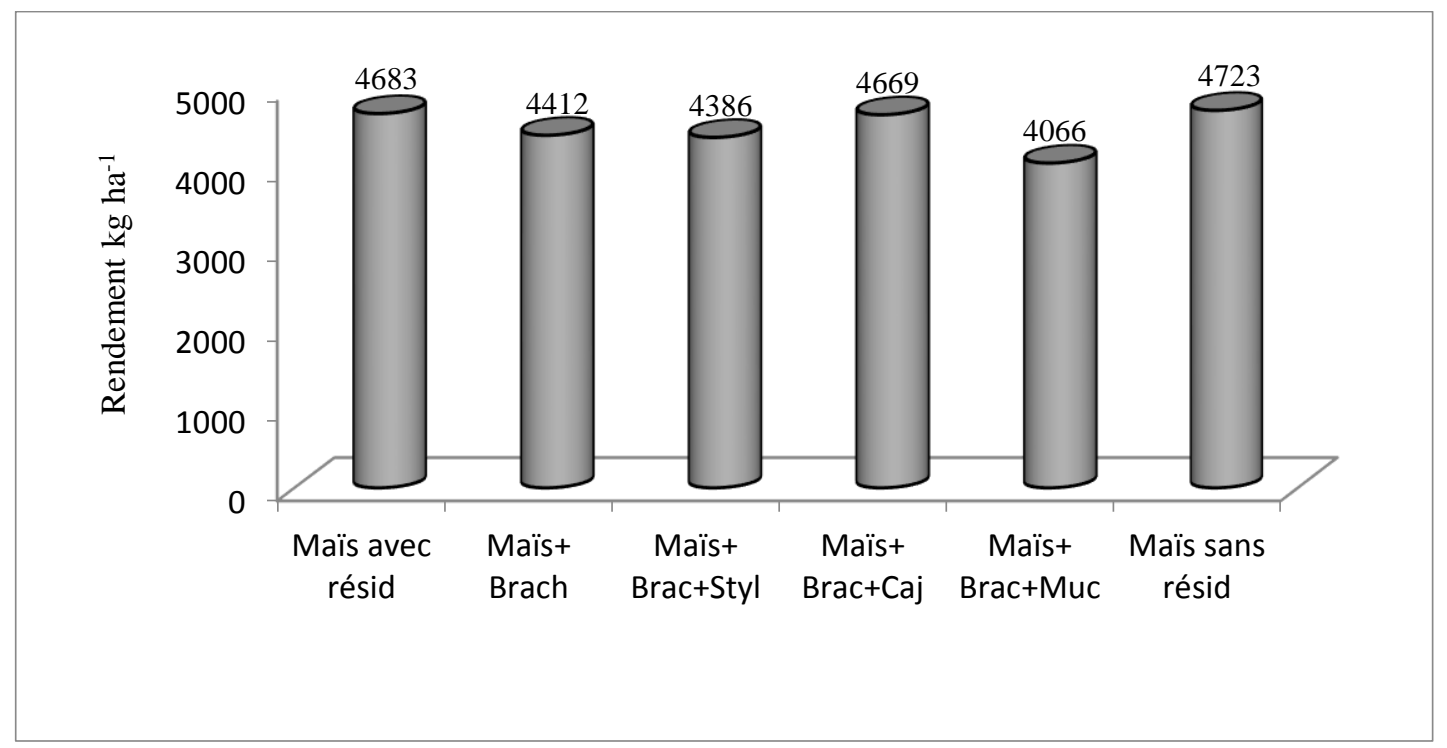

Légende $:$ Résid = résidus de récolte $;$ Brach = Brachiaria ruziziensis $;$ Caj = Cajanus cajan $;$ Muc = Mucuna sp

Figure 3 : Rendements moyens du maïs dans les différents systèmes de culture à la station de recherche agronomique de Sotuba de 2014 à 2018. 
Tableau 5 : Nombre d'Unité de Bétail Tropical pouvant être supplémenté pendant 90 jours.

\begin{tabular}{lccccc}
\hline \multirow{2}{*}{ Systèmes de culture } & \multicolumn{3}{c}{ UB T } \\
\cline { 2 - 6 } & $\mathbf{2 0 1 4}$ & $\mathbf{2 0 1 5}$ & $\mathbf{2 0 1 6}$ & $\mathbf{2 0 1 7}$ & $\mathbf{2 0 1 8}$ \\
\hline T1 : maïs semé directement sur sol nu sans labour & $6,33 \mathrm{~b}$ & $6,02 \mathrm{~b}$ & $6,69 \mathrm{~b}$ & $6,59 \mathrm{~b}$ & $12,48 \mathrm{a}$ \\
T2= maïs + Brachiaria ruziziensis & $7,89 \mathrm{~b}$ & $8,51 \mathrm{ab}$ & $8,39 \mathrm{ab}$ & $8,23 \mathrm{ab}$ & $7,78 \mathrm{~b}$ \\
T3= maïs + Brachiaria ruziziensis + Stylosanthes hamata & $8,01 \mathrm{ab}$ & $10,34 \mathrm{ab}$ & $8,17 \mathrm{ab}$ & $8,53 \mathrm{ab}$ & $9,82 \mathrm{ab}$ \\
T4= maïs + Brachiaria ruziziensis + Cajanus cajan & $9,72 \mathrm{ab}$ & $12,77 \mathrm{ab}$ & $9,98 \mathrm{ab}$ & $8,73 \mathrm{ab}$ & $8,38 \mathrm{ab}$ \\
T5= maïs + Brachiaria ruziziensis + Mucuna sp & $12,09 \mathrm{a}$ & $13,66 \mathrm{a}$ & $13,73 \mathrm{a}$ & $13,68 \mathrm{a}$ & $9,32 \mathrm{ab}$ \\
T6= maïs seul (exportation des résidus après la récolte : & & & & \\
système conventionnel) & $6,39 \mathrm{~b}$ & $7,06 \mathrm{ab}$ & $5,59 \mathrm{c}$ & $6,04 \mathrm{~b}$ & $10,40 \mathrm{ab}$ \\
\hline Ecart type & 0,48 & 0,09 & 0,05 & 0,11 & 0,15 \\
CV (\%) & 8,28 & 2,85 & 4,08 & 5,01 & 3,04 \\
Probabilité & 0,008 & 0,001 & 0,000 & 0,000 & 0,006 \\
\hline
\end{tabular}

Les lettres indiquent la différence entre les traitements.

Tableau 6 : Quantité de fumure organique estimée avec les UBT en stabulation pendant 90 jours utilisant la biomasse produite dans les différents systèmes de culture.

\begin{tabular}{lccccc}
\hline \multirow{2}{*}{ Systèmes de culture } & \multicolumn{4}{c}{ Fumure organique } \\
\cline { 2 - 6 } & $\mathbf{2 0 1 4}$ & $\mathbf{2 0 1 5}$ & $\mathbf{2 0 1 6}$ & $\mathbf{2 0 1 7}$ & $\mathbf{2 0 1 8}$ \\
\hline$\cdot$ & $1584 \mathrm{~b}$ & $1504 \mathrm{~b}$ & $1672 \mathrm{a}$ & $1648 \mathrm{~b}$ & $3120 \mathrm{a}$ \\
T2= maïs + Brachiaria ruziziensis & $1972 \mathrm{ab}$ & $2128 \mathrm{ab}$ & $2097 \mathrm{ab}$ & $2058 \mathrm{ab}$ & $1944 \mathrm{~b}$ \\
T3= maïs + Brachiaria ruziziensis + Stylosanthes hamata & $2002 \mathrm{ab}$ & $2585 \mathrm{ab}$ & $2044 \mathrm{ab}$ & $2133 \mathrm{ab}$ & $2456 \mathrm{ab}$ \\
T4= maïs + Brachiaria ruziziensis + Cajanus cajan & $2429 \mathrm{ab}$ & $3193 \mathrm{a}$ & $2496 \mathrm{ab}$ & $2183 \mathrm{ab}$ & $2095 \mathrm{ab}$ \\
T5= maïs + Brachiaria ruziziensis + Mucuna sp & $3022 \mathrm{a}$ & $3414 \mathrm{a}$ & $3433 \mathrm{a}$ & $3420 \mathrm{a}$ & $2331 \mathrm{ab}$ \\
T6= maïs seul (exportation des résidus après la récolte : & $1599 \mathrm{~b}$ & $1764 \mathrm{~b}$ & $1397 \mathrm{~b}$ & $1509 \mathrm{~b}$ & $2601 \mathrm{ab}$ \\
système conventionnel) & & & & & \\
\hline Ecart type & 5,28 & 0,06 & 0,07 & 0,16 & 0,08 \\
CV (\%) & 0,04 & 0,02 & 0,03 & 0,00 & 0,06 \\
Probabilité & & & & & \\
\end{tabular}

Les lettres indiquent la différence entre les traitements.

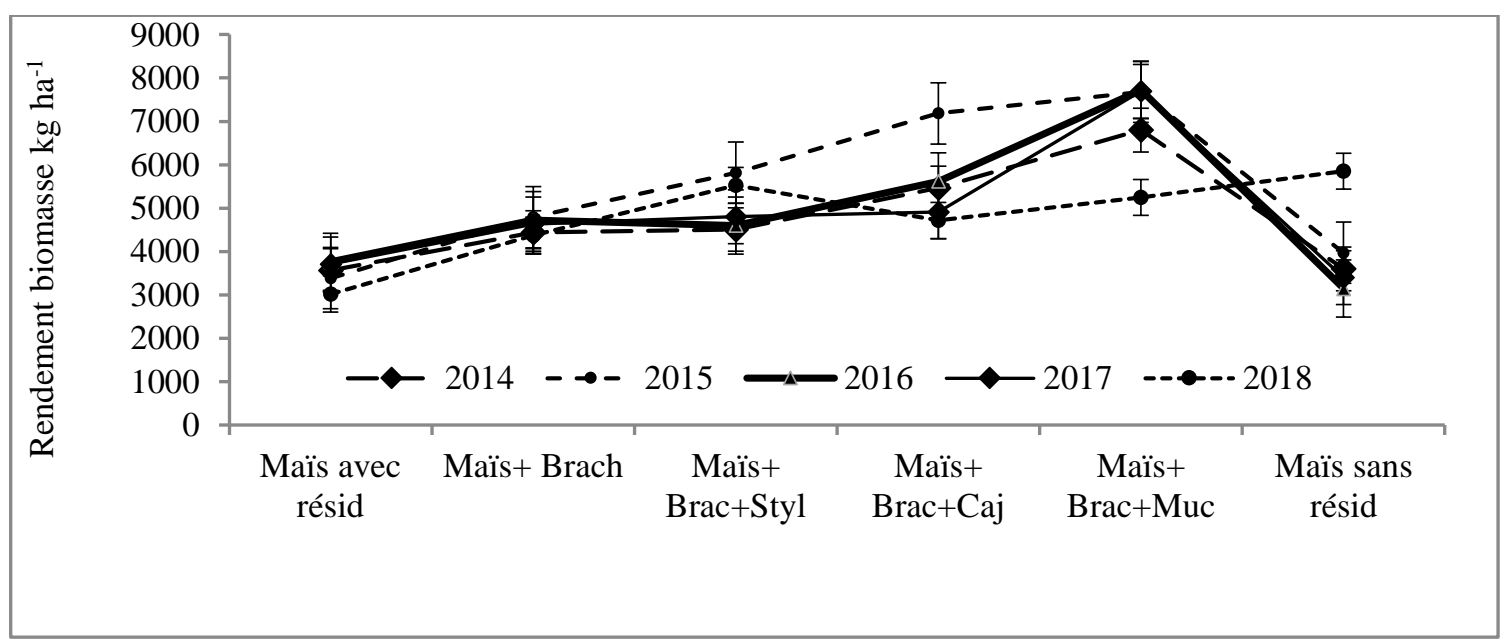

Figure 4 : Rendement de biomasse fourragère totale annuelle dans les différents systèmes de culture de 2014 à 2018 à la Station de Sotuba. 


\section{DISCUSSION}

La densité recommandée pour la culture du maïs est de 62500 plants par hectare. Les densités de peuplement du maïs obtenues à la récolte dans le cadre de notre étude ont été inférieures à la densité théorique de 62500 plants par l'hectare (51300 plants/ha pour les parcelles avec résidus et 52300 plants par hectare pour les parcelles sans résidus). Ce résultat est supérieur à celui trouvé par Coulibaly et al. (2017) qui ont montré dans une expérimentation conduite au Burkina Faso pendant trois campagnes que malgré les ressemis les densités de peuplement du maïs était compris entre 12374 plants par hectare et 36507 plants par hectare. Au Mali, Coulibaly et al. (2017) ont observé après deux années d'expérimentation des densités similaires. Au Cameroun, Guibert et al. (2016) ont obtenu à la récolte des densités comprises entre 27400 et 40000 plants par hectare pour une densité recommandée de 50000 plants par hectare. Des pertes de plants ont également été constatées entre le comptage réalisé après le démariage et la récole. Les travaux de Bayer (2014) ont montré des pertes de 5 à $8 \%$ de plants entre le nombre de plants au semis et le nombre de plants à la récolte.

Nos résultats ont montré que l'insertion des plantes de couverture dans les systèmes de culture du maïs n'a pas d'effet négatif sur la croissance du maïs. Ce résultat est similaire à celui obtenu par Coulibaly et al. (2017). Dans l'expérimentation, la production moyenne de biomasse était comprise entre $3143 \mathrm{~kg} \cdot \mathrm{ha}^{-1}$ en culture pure du maïs à $7724 \mathrm{~kg} \cdot$ ha $^{-1}$ en culture associée maïs + Brachiaria ruziziensis + Mucuna cochinchinensis. La production de biomasse ainsi obtenue permet de supplémenter entre 6,02 et 13,73 UBT pendant 90 jours. La production de biomasse dans les pâturages naturels a une valeur maximale de 10,6 t.MS.ha ${ }^{-1}$ dans les formations rizicoles et un minimum de 2,9 t.MS.ha ${ }^{-1}$ dans les savanes arborées denses (Sanon et al., 2014). Selon la même source, les savanes arbustives et arborées claires ont des productions intermédiaires. L'insertion des plantes de couverture dans les systèmes de culture permet d'améliorer la production de fourrage. Coulibaly et al. (2017) ont obtenu en milieu réel en culture pure de maïs une production de biomasse de $5405 \mathrm{~kg} \mathrm{MS}$.ha ${ }^{-1}$, en association

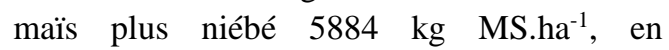
association maïs plus mucuna $4113 \mathrm{~kg}$ MS.ha $^{-1}$. Ils ont également montré que la production de biomasse de

légumineuses améliore le bilan fourrager et permet de couvrir les besoins de matières sèches des animaux des exploitations agricoles. L'insertion des plantes de couverture dans les systèmes de culture permet donc d'améliorer la production de fourrage et renforcer l'intégration agriculture élevage des exploitations agricoles.

La biomasse sèche obtenue dans l'expérimentation peut produire entre 1504 et $3433 \mathrm{~kg}$ de fumure organique pendant trois mois. En fonction de la supplémentation des animaux, la production de fumure organique peut varier entre 58 et $813 \mathrm{~kg}$ MS.UBT $^{-1}$ (Bénagabou et al., 2017).

Les rendements obtenus sont compris entre 3508 et 5469 kg.ha-1. Ils ont été supérieurs à ceux obtenus par Coulibaly et al. (2017) en milieu paysan avec la même variété de maïs (2600 kg/ha). Guibert et al. (2016) ont obtenu des rendements compris entre 1,3 t/ha et 2,3 t/ha sur des sols dégradés et peu fertiles. $\mathrm{Au}$ Cameroun des rendements en maïs graine de 2,38 t/ha et 2,44 t/ha ont été obtenus par Nchoutnji et al. (2010) dans les conditions de culture associée avec le Mucuna. Les résultats de nos expérimentations montrent que l'association maïs culture associée ne réduit pas les rendements grains du maïs et augmente les quantités totales de biomasse produites, nécessaires à l'alimentation du bétail

\section{Conclusion}

La situation pluviométrique a varié dans le temps et dans l'espace. Le démarrage de la campagne a souvent été difficile, mais le cumul a été supérieur au besoin du maïs toutes les années de l'expérimentation.

L'insertion des plantes de couverture n'a pas affecté le rendement du maïs. La production de biomasse a été suffisante pour 
alimenter 6 à 13 UBT pendant trois mois. Une quantité importante de fumure organique peut être produit quand les animaux sont en stabulation. Cette fumure organique produite joue un rôle très important dans le maintien de la fertilité des parcelles. L'association maïsculture associée (Brachiaria, Мисиna) ne réduit pas les rendements grains du maïs et augmente les quantités totales de biomasse produites, nécessaires à l'alimentation du bétail. L'innovation ainsi testée peut-être proposée aux producteurs des différentes zones agro écologiques du Mali.

\section{CONTRIBUTIONS DES AUTEURS}

Les collègues co-auteurs ont participé à la collecte des données et ont également contribué à l'analyse des données.

\section{CONFLIT D'INTÉRÊTS}

Les auteurs déclarent qu'ils n'ont aucun conflit d'intérêts pour cet article.

\section{REMERCIEMENTS}

Les auteurs remercient la Coopération Brésilienne et les pays partenaires du Projet Coton $\mathrm{C} 4+\mathrm{T}$ chad. Ce travail a été soutenu financièrement par l'Agence Brésilienne de Coopération $(\mathrm{ABC})$ à travers l'Ambassade Brésilienne au Mali.

\section{REFERENCES}

Balarabé O. 2012. Capital Sol et arrangements institutionnels dans les agrosystèmes du Nord-Cameroun. Thèse doctorant, SupAgro, Montpellier, $213 \mathrm{p}$.

Bayer 2014. Optimiser son semis de maïs pour un meilleur rendement de maïs à l'hectare. https://www.dekalb.fr/maisgrain/conseils-pour-planter-et-cultiverle-mais/semis-de-mais/rendement-dumais-grain, $4 \mathrm{p}$.

Bénagabou OI, Blanchard M, Bougouma/Yaméogo VMC, Vayssières $\mathrm{J}$, Vigne M, Vall E, Lecomte P, Nacro HB, 2017. Does crop-livestock integration improve energy-use efficiency, recycling and self-sufficiency of smallholder farming systems in
Burkina Faso? Rev. Elev. Med. Vet. Pays Trop., $\quad$ 70(2): $\quad 31-41 . \quad$ DOI: 10.19182/remvt.31479

Blanchard M (2010). Gestion de la fertilité des sols et rôle du troupeau dans les systèmes coton-céréales-élevage au Mali-Sud. Savoirs techniques locaux et pratiques d'intégration agriculture élevage. Thesis, $\mathrm{PhD}$. Universite Paris-Est, Creteil, Val De Marne.

Coulibaly D, Sissoko F, Doumbia S, Ba A, Dembélé D. 2017. Evaluation de l'effet de la fertilisation minérale sur la production des variétés améliorées de maïs et le disponible fourrager en zone cotonnière du Mali-Sud (Mali). Agronomie Africaine Sp., 29(1) : 109-117

Coulibaly K, Gomgnimbou APK, Traoré M, Nacro HB, Sedogo M.P. 2017. Effets des associations maïs-légumineuses sur le rendement du maïs (Zea mays L.) et la fertilité d'un sol ferrugineux tropical à l'Ouest du Burkina Faso. Afrique SCIENCE, 13(6): 226-235. http://www.afriquescience.info

Deveze JC, Halley des Fontaines D. 2005. Débats, Echanges, Réflexions en Réseaux Développement Rural au Sud. Document AFD : Paris.

FAO. 2015. Mesurer la Durabilité des Systèmes de Culture du Coton. Organisation des Nations Unies pour l'alimentation et l'agriculture Comité consultatif International du coton. Rome, 2015 ; p 177.

Fleury M. 2016. Agriculture itinérante sur brûlis (AIB) et plantes cultivées sur le haut Maroni : étude comparée chez les Aluku et les Wayana en Guyane Française, Brasil. Boletim do Museu Paraense Emílio Goeldi. Ciências Humanas, 11(2) : 431-465. DOI : http://dx.doi.org/10.1590/1981.81222016 000200006

Guibert H, Kenne Kueteyim P, Olina Bassala J-P, M'Biandoun M. 2016. Intensifier la culture du maïs pour améliorer la sécurité alimentaire : le producteur du Nord 
Cameroun y a-t-il intérêt ? Cah. Agric., 25 : 65006. DOI : 10.1051/cagri/2016048

Koné A. 2013 : Impacts socioéconomiques de l'utilisation de la jachère forestière améliorée pour la récupération des terres salées dans la région de Kaolack, au Sénégal, Maîtrise en agroforesterie, Québec, $141 \mathrm{p}$.

Lemaire G. 2014. L'intégration AgricultureElevage, un enjeu mondial pour concilier production agricole et environnement. Innovations Agronomiques, 39 : 181-190

Menyonga JM, Bezuneh T, Yayock JY, Soumana I. 1994. Progress in food grain research and production in semi-arid Africa. In: Proceedings of the SAFGRAD Inter-Network Conference, Niamey, Niger, 7-14 march 1991. Ouagadougou (Burkina Faso): OAU/STRCSAFGRAD. [Google Scholar].

Nchoutnji I, Dongmo, AL, Mbiandoun M, Dugué P. 2010. Accroître la production de la biomasse dans les terroirs d'agroéleveurs : cas des systèmes de culture à base de céréales au Nord Cameroun. Tropicultura, 28 (3) : 133-138

Odru M. 2013. Flux de biomasse et renouvellement de la fertilité des sols à l'échelle du terroir. Etude de cas d'un terroir villageois sereer au Sénégal. Mémoire de fin d'études. Ecole Supérieure d'Agro-Développement International. Dakar, Sénégal, p. 109 p.
Sanon HO, Avadogo M, Tamboura HH, Kanwé BA. 2014. Caractérisation des systèmes de production et des ressources fourragères dans un terroir test de la zone soudanienne du Burkina Faso. Vertigo, la revue électronique en sciences de l'environnement, $\quad \mathbf{1 4}(2) . \quad$ DOI : https://doi.org/10.4000/vertigo.15171

Sissoko F, Diarra S, Traoré M. 2020. Le semis direct sous couverture végétale : une opportunité de mise en place rapide du cotonnier en culture pluviale au Mali. Int. J. Biol. Chem. Sci., 14(3): 722-738. DOI: https://doi.org/10.4314/ijbcs.v14i3.7

Sissoko F, Autfray P. 2007. Projet PASE SCV Rapport d'activités 2006. IER/CIRAD Mali.

Thibaud B, François A. 2010. Systèmes de production et durabilité dans les pays du Sud. In Enjeux de la Durabilité des Systèmes de Production dans les Suds. Editions Karthala ; 120-131. http///www.karthala.com.

Vall E, Andrieu N, Chia E, Nacro HB, 2012. Intégration agriculture-élevage et intensification écologique dans les systèmes agrosylvopastoraux de l'Ouest du Burkina Faso, province du Tuy. Actes du séminaire ASAP, novembre 2011, Bobo-Dioulasso, Burkina Faso. 